\title{
Precision Mechanics of Contact Surfaces in the Transition From Stick to Slip
}

\author{
SHALAPKO Jurji ${ }^{1, a}$, RADEK Norbert ${ }^{2, b}$, PIETRASZEK Jacek ${ }^{3, c}$
}

${ }^{1}$ University of Technology and Life Sciences in Bydgoszcz. , ul. Fordońska 430, 85-790

Bydgoszcz, Poland

${ }^{2}$ Centre for Laser Technologies of Metals, Technical University of Kielce

Al. 1000-lecia Państwa Polskiego 7, 25-314 Kielce, Poland

${ }^{3}$ Institute of Applied Informatics, Cracow University of Technology

Al. Jana Pawła II 37, 31-864 Kraków, Poland

ashalapko@yahoo.com, ${ }^{b}$ norrad@tu.kielce.pl, ${ }^{c}$ pmpietra@mech.pk.edu.pl

Keywords: contact mechanics, stress, stick-slip, fretting.

\begin{abstract}
The article deals with the problem of transition quasi-static contact in global slip under cyclic tangential load. The state of stress in partial contact, the laws of friction and scenarios of transition to slip of metal surface was determined. Partial slip is defined by the existence of zones stick and slip within the contact area. Currently, there are two basic concepts concerning surface damage processes: one connected with surface activation, which involves an increase in free energy in tribological system, and the other, connected with surface passivity, when free energy decreases. Experimental and theoretical studies were conducted to determine the contact between a sphere and a plane, which is the most suitable system for simulating of small-amplitude fretting $(\sim 0-3$ microns).
\end{abstract}

\section{Introduction}

The relative displacement at small amplitude of the two surfaces in contact leads to the development of damage and even to fretting-wear. This phenomenon is called fretting.

Phenomenon of fretting occurs in contact between components that are together and receives the cyclic loading with small amplitude that causes them to some small tangential displacement relative to each other. Fretting damage is often the origin of catastrophic failures or loss of functionality in many industrial applications, Considered as a problem for modern industry, fretting is encountered in all cases of quasi-static loadings submitted to vibration and hence many industrial branches. Specifically, fretting damage to occur in parts found in helicopters, fixed-wing aircraft, trains, ships, automotives, trucks and buses, farm machinery, engines, construction equipment, orthopaedics implants, artificial hearts, rocket engine, wire ropes, etc. Fretting is now fully identified as a small amplitude oscillatory motion which induces a harmonic tangential force between two solid surfaces in contact, which units in self much the dynamic and tribological phenomena in the contact [1-3]. Similar phenomena may occur in contact precision sensors $[4,5]$. Similar phenomena may occur in contact precision sensors. Under the influence of the normal load and external of vibration field of electromagnetic radiation is disruption of sensor work. The role of vibration frequency on the fatigue behaviour for the ductile Iron is shown in a recent study [6].

Recently increasing attention of the researchers is given to quasistatic contact in conditions of dynamic loading. It is possible to state, that the given condition of contact is characteristic in most cases where really operate of nominal - fixed joints, when there is no visible relative displacement $[7,8]$. The last statement requires detail discussion with the purpose of definition of parameters of small amplitude of fretting. The difficulties consist that for specific state of stress - strain of surface, an amplitude-frequency characteristic of oscillations of two bodies practically is identical, that, a priori excludes, relative displacement of surfaces. However, inspection of the interface of surface indicates the damages as characteristic for fretting. It is possible to assume, that the elastic interaction of two bodies in a tangential direction suffices for initiation fretting. In this case 
configuration of separate spots of contact both in scale of roughness, well as in the boundaries of contour area of contact allows initiating relative micro displacement up to several microns. It is enough of it for intensive evolution of fretting in the boundaries of a preliminary displacement of surfaces (Fig. 1).
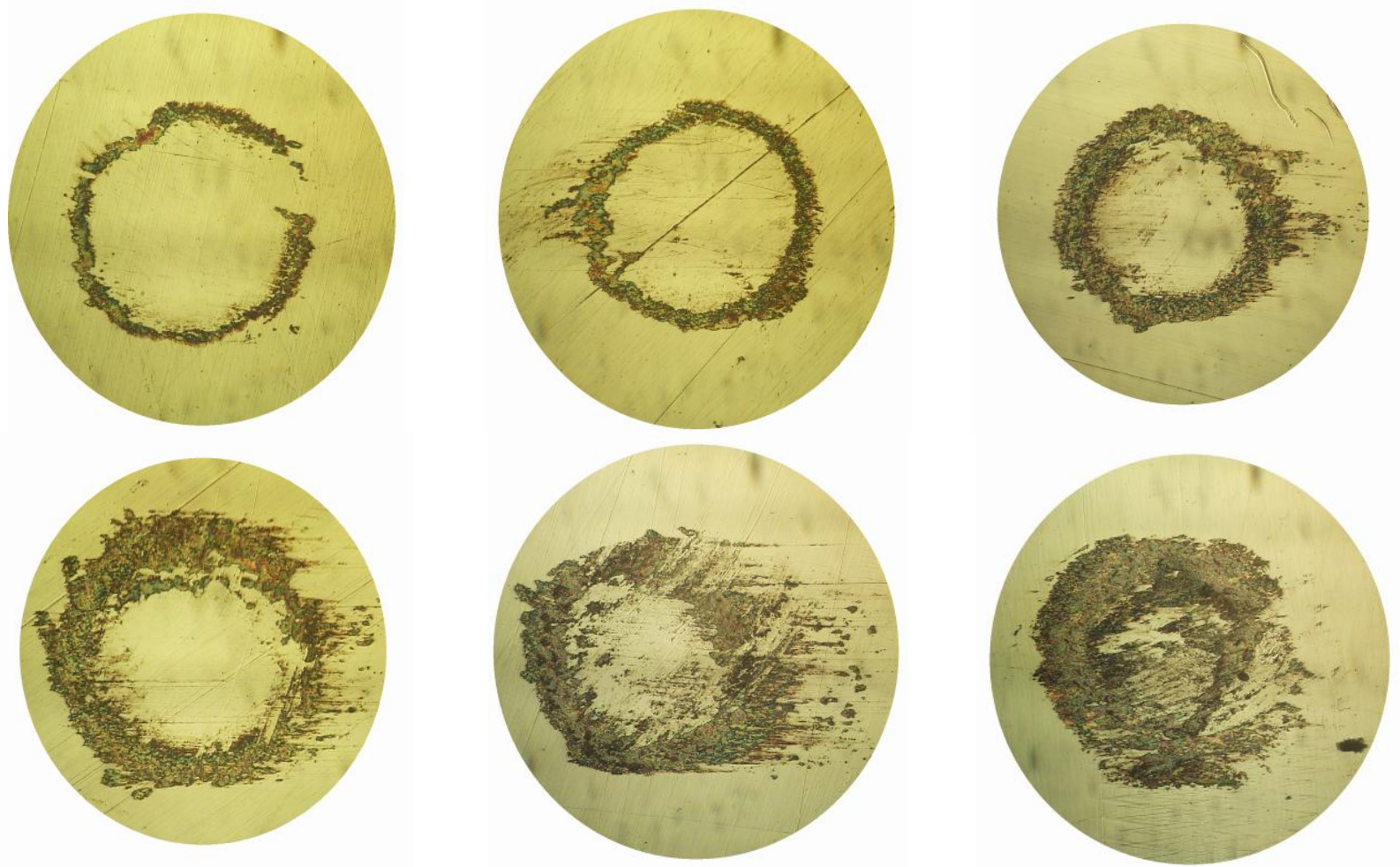

Fig.1. Evolution of geometry and damage of the contact spot for flat surface of carbon steel and steel ball (diameter $12.7 \mathrm{~mm}$ ) in the transition from the stick to slip.

Indirect measurement of the amplitude of fretting as a displacement difference between the samples in many cases, are not in accordance with the actual slip. And this discordance is more, than less amplitude of a tangential displacement. A complex effect of an adhesion, elasticity of a contact layer and dynamic phenomena into a system "contact - subsurface layer - surface as a whole", do not give accurate significance of slip amplitude by usual method of a measurement. Our interest for friction has three main principles: the law of friction with low velocities, contact dynamics as a whole and determination of the latent features for evolution of surface contact at cyclic and tangential vibration.

The relative velocity of surfaces, under the micro slip of surfaces, changes significantly in a very short time (from zero up to several hundred microns / s) and, with another side, the relative displacement almost is constant (may be in borders of one micron). Action by micro slip occurring at the interface of two bodies and occurs of a jump dynamic properties of the contact system. The overall, process of friction describes as a balance three factors: the work has done by the friction force, kinetic energy and potential energy of elastic-plastic deformation of the surface layer.

The evolution of the system can occur under different scenarios, but in general, tends to increase the energy of the system, as display of the growth the kinematic factor (increased amplitude slip) and reduction potential energy (elastic of contact deformation).

\section{Stress-strain state of nominal-fixed frictional contact (NFFC)}

As has been demonstrated, damage changes the distribution of the dynamic picture of NNFC due to a combination wear areas for elastic surface and of contact fatigue in the elastic-plastic area (Fig. 2). We define the components of stresses in the half-space caused by of the tangential load of contact (Fig.3). 


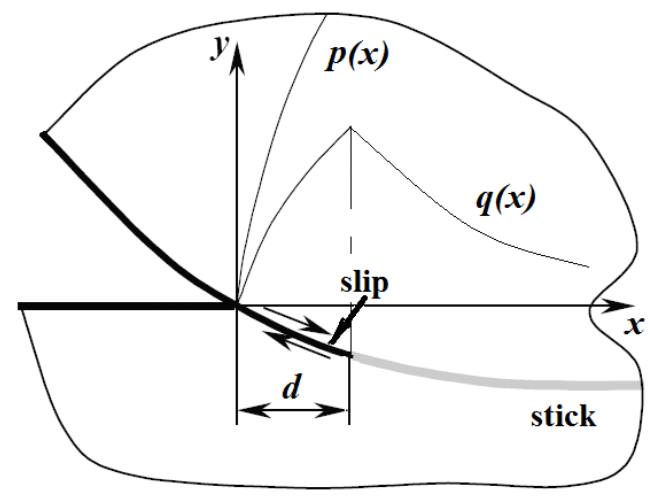

Fig. 2. The distribution of normal stresses $p(x)$ and tangential $q(x)$ in contact of ball with flat surface.

The circular contact area of ball and half-space at compressive by normal force $P_{n}$ according to the formula given by Hertz:

$$
a=\left(\frac{3}{4} \cdot \frac{\left(1-v^{2}\right) \cdot P_{n} \cdot R}{E}\right)^{\frac{1}{3}}
$$

where:

$v$ - Poisson's coefficient;

$P_{n}$ - normal load;

$R$ - radius of the sphere;

$1 / E=\left(1-v^{2}\right) / E_{1}+\left(1-v^{2}\right) / E_{2}-$ Young's modulus.

Tangential stress which acting along the axis according to law Amontons-Coulomb:

$$
q(x)=\frac{3 \mu \cdot P_{n}}{2 \pi a^{3}}\left(a^{2}-x^{2}\right)^{\frac{1}{2}}
$$

The distribution of normal stress:

$$
\sigma(x)=\frac{3 P_{n}}{2 \pi a^{2}}\left(a^{2}-x^{2}\right)^{\frac{1}{2}}
$$

Laws of stress distribution and geometric parameters of the contact are shown in Figure 3.

The hysteretic nonlinear dependence of sliding process of contact surface for states of the normal loading, pre-sliding friction (growth of joints and partial slip on periphery of spots of contact) and gross sliding for all spots of contact in the proper stages presented on Fig.4.
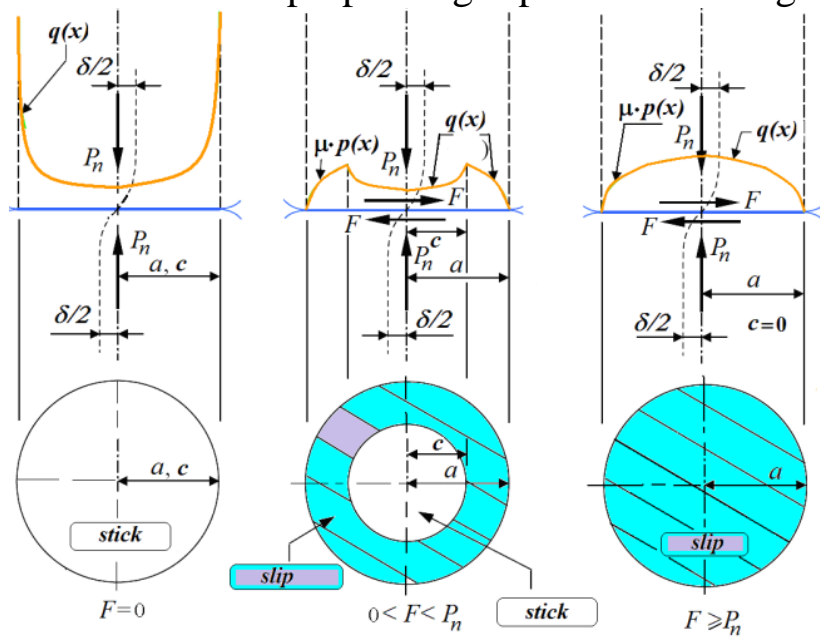

Fig. 3. The overall picture of the evolution of the stress state and geometry of contact spots at the transition from absolute stick to gross slip 

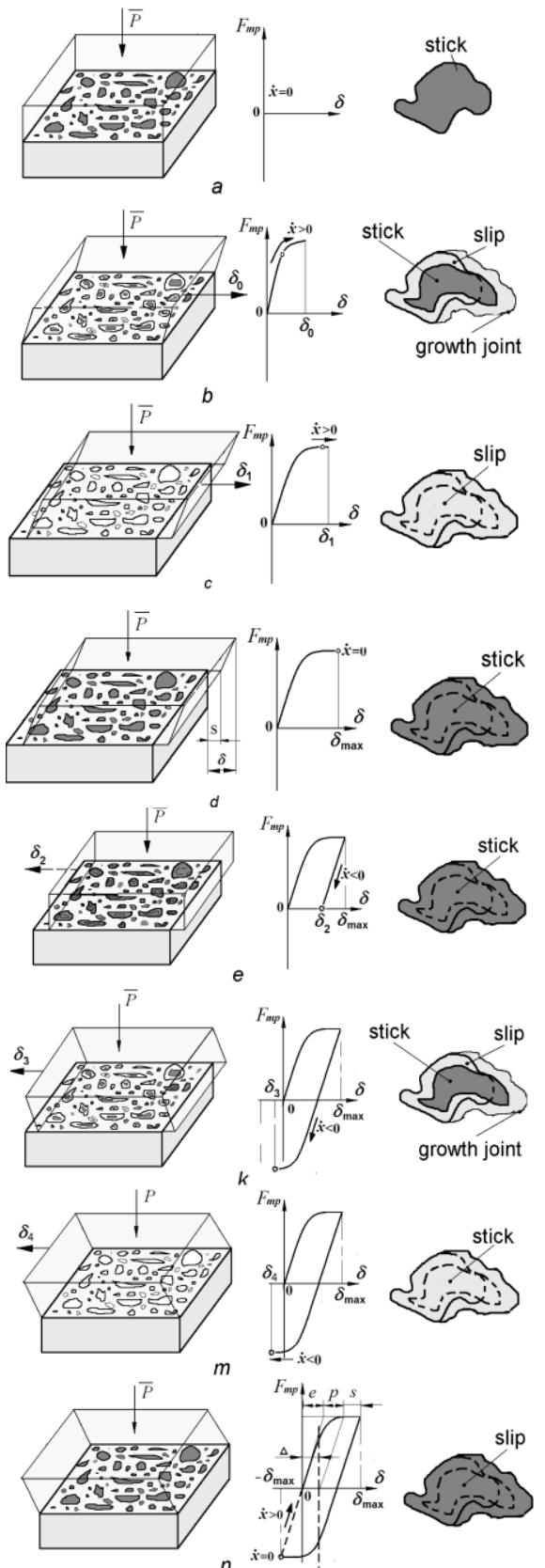

Fig.4. Mechanics of multicontact of interface in the direction of tangential loading with global sliding

The stages of mechanical interaction of discrete contact can be described by the following stages (Fig.3): a - normal loading on rough surface , $\mathrm{b}$ - state of pre-sliding, $\mathrm{c}$ - global slip on all spots of contact, $\mathrm{d}-$ state of stop in extreme right position, $\mathrm{e}-$ fall of the tangential loading to the zero and formed exclusively the normal contact, $\mathrm{k}$ - state of pre-sliding, $\mathrm{m}$ - global slip on all spots of contact, $\mathrm{n}$ - moment of stop in extreme position, beginning of decreasing of friction force and setting of the initial state of interface.

At the long time cyclic loadings every spot of contact has its evolution of development and carries the part of general force of friction, which save of nominally integrity of contact. In this connection we will suppose that creation of artificial spots of contact which would differ by large resistive capacity to destruction in tangential direction will enable to increase fretting - resistance of nominally fixed joints. 
Assume that the size of the contact and distribution efforts from the normal force $P_{n}$ is independent of the application in the future tangential force $F$. Given all the assumptions about of size of contact spots, contact stresses, independence of the total volume load and fixing details, lack of normal forces outside the contact, form the task as follows: necessary to determine the distribution of contact stresses on the surface and equivalent stresses into half space under conditions of partial slip in NFFC and the transition to a global sliding surfaces. The boundary conditions for half space at the static loading set the radius $c$, normal stress $\sigma(x)$ and related tangetional efforts $q(x)= \pm \mu \sigma(x)$. Outside the contact surface is free from stress. Geometric parameters of contact with the stick and slip zone determined by the known formula Mindlin [7]:

$$
c / a=\left(1-F / \mu P_{n}\right)^{1 / 3}
$$

Expression (4) shows a general upward trend in the area of sliding contact. However, it does not reflect the important parameters of Friction, determining dynamic phenomena in contact. Experimental studies have shown, that the transition to sliding occurs at proper of minimum ratio $\xi_{\min }=c / a$. This establishes the fact that laws of static friction $F=\mu P_{n}$ in the formula (4) limited to some critical values of tangential force $F_{\text {critical }}$. You must specify the coefficient of friction and take it as for the static contact where the keep the integrity at maximum shear strength $F_{\max }$. Thus, taking into account the pulse shape of the friction force and the maximum force of static friction, Mindlin formula specified as follows:

$$
\xi(t)=c / a=\left(1-F(t) / F_{\max }\right)^{1 / 3}
$$

Depending on the law of friction can be a fundamental change in the state of NFFC. Let the friction force increases cinematically from zero time in four laws:

- energy of friction law $F(t)=k_{1} \cdot t^{2}$;

- proportional to the time $F(t)=k \cdot t$;

- sinusoidal $F(t)=F \cdot \sin (\omega t)$;

- Person's law $F(t)=F \cdot\left(1-e^{-\frac{t}{\tau}}\right)$, where $k, k_{1}, \tau$ - permanent; $\omega$ - circular frequency [8].

Figure 5 shows the results of the calculation. Note two things: under the Person's law of contact saves the state of the nominal grip for longer than other laws. Due to minor fluctuations in friction, which cannot be predicted, we again meet with the instability of the system depending on the small parameter changes. Transition to slip theoretically occurs at very short intervals, especially laws 1 and 2 at the end of the first quarter period.

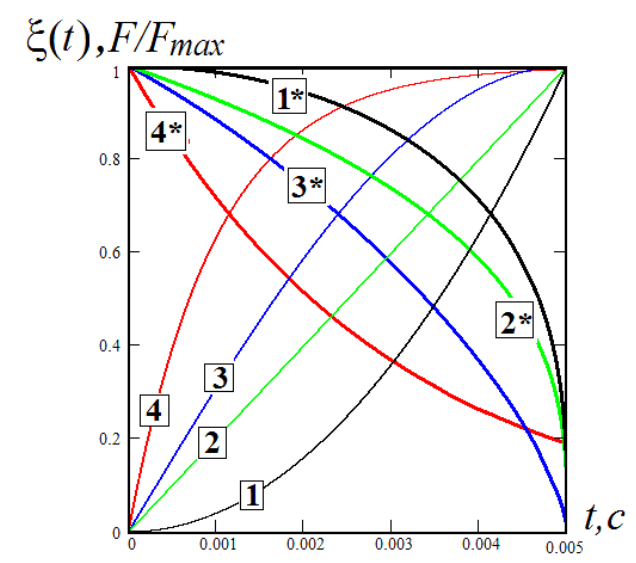

Fig. 5. The transformation of ratio of stick and slip area

(in fig. marked $*$ ) in NFFC depending on friction force. 
Here laws of friction:

$$
\begin{aligned}
& 1-F(t)=k_{1} \cdot t^{2}, k_{1}=6 \cdot 10^{5} \\
& 2-F(t)=k \cdot t, k=3 \cdot 10^{3} \\
& 3-F(t)=F \cdot \sin (\omega t), \omega=628 \mathrm{rad} / \mathrm{s} \\
& 4-F(t)=F \cdot\left(1-e^{-t / T}\right), \tau=0,001 .
\end{aligned}
$$

The role of small parameter of friction force on the duration stays in the state of sticking of contact

Numerous studies of contact spots have shown that under the same load conditions the transition from stick to slip occurs at different values of the ratio $\xi$. The discrepancy reaches to $50 \%$. This can be explained by several factors: randomness of contact and dynamic friction at a time. Simulating the behaviour of the system at approaches the force of static friction to maximum (Fig. 6).

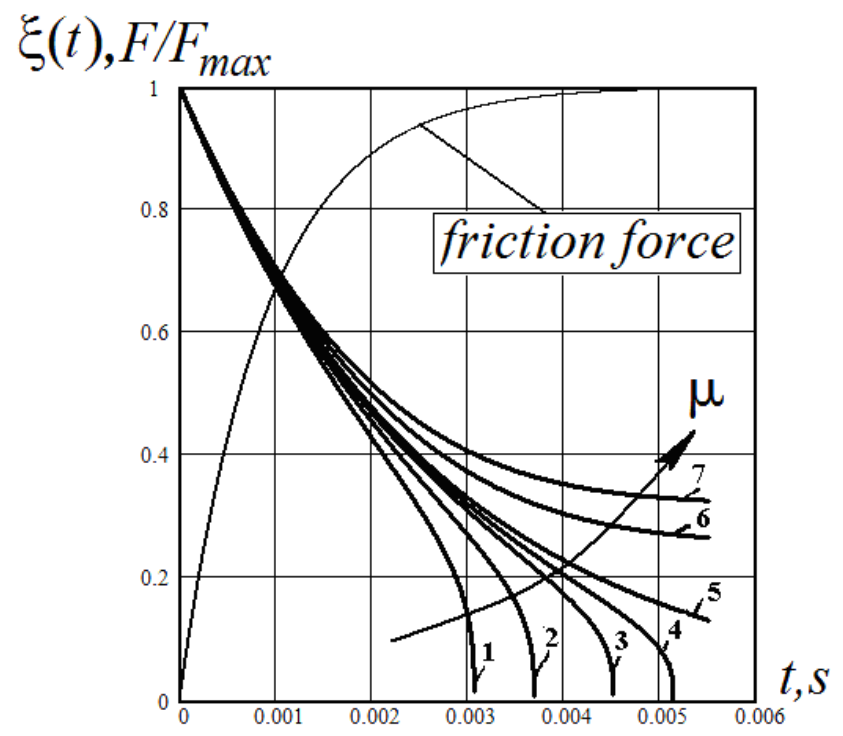

Fig.6. The influence of small changes in the coefficient of friction on ambiguity at the transition the partial slip to full slip $F_{\max }=0.3 \cdot P_{n}: 1-\mu=0.29 ; 2-0.295 ; 3-0.297 ; 4-0.299 ; 5-0.3 ; 6-$ $0.305 ; 7-0.31$

At the moment of achievement ratio $\xi(t)$ zero the zone of stick disappears. Small change in the parameter, in our case, the coefficient of friction, can dramatically change the state NFFC.

\section{Conclusions}

Depending on the kinematics of increase strength in shear (that frequency of the perturbation), formed of friction of two surfaces (history of loading) and ratio of zones of slip and stick is drastically different.

Dimensions slip zone is very sensitive to changes into the friction. We can conclude that for each pair of contact exists own way of formation of stick-slip areas in which it transition to full slip. This is not a consequence of phenomenon of slow increase of area of slip and a corresponding decrease in the contact spot in the moment of full slip.

Given, that traditional frequency with which work of NFC is quite small, it was determined that a joint residence of contact elements in a state of presiding is within 0.0075 ... 0.015 seconds. So, given micro geometry contact surfaces, increase connections, elastic-plastic characteristics, frequency perturbation can determine the characteristic of micro velocity in the characterization of friction at which the transition from absolute rest to a relative displacement of the two surfaces. 


\section{Reference}

[1] J.I. Shalapko, S.G. Kostogryz, Research process of fretting-corrosion in nominal joint by modeling variation pressure, in: Proc. of Conf. Tribological Problems in Exposed Friction Systems, Bratislava, Slovak Republic, 2002, pp. 292-296.

[2] J.I. Shalapko, S.G. Kostogryz, Evolution of stick-slip regime and chaos for fretting-process, in: Proc. of $8^{\text {th }}$ Conf. Active noise and vibration control methods, Krasiczyn, Poland, 2007, pp. $432-443$.

[3] V.S. Kurskoy, J.I. Shalapko, N. Radek, Modeling of dynamical behavior of nominal-fixed joints, in: J.I. Shalapko, L.A. Dobrzański (Eds.) Scientific basis of modern technology: experience and prospects. Gliwice, Poland, 2011, pp.319-332.

[4] L. Radziszewski, The influence of the surface load exerted by a piezoelectric contact sensor on testing results: Part II, The electrical transients generated by piezoelectric sensor, Archives of Acoustics 28 (2003) pp.93-100.

[5] L. Radziszewski, Intrusive effect of a contact transducer on testing results, Metrology and Measurement Systems Vol. XI, 1/2004, pp.31-43.

[6] R. Ulewicz, F. Novy, J. Selejdak, Fatigue Strength of Ductile Iron in Ultra-High Cycle Regime, Advanced Materials Research 874 (2014) 43-48.

[7] R.D. Mindlin, Elastic spheres in contact under varying oblique forces, ASME J. Appl. Mech. 75 (1953) pp.327-344.

[8] B.N.J. Persson, Sliding Friction Physical Principles and Applications, Springer, Berlin, 2000. 